\title{
An integrable hierarchy with a perturbed Hénon-Heiles system
}

\author{
A.N.W. Hone ${ }^{*}$ V. Novikov ${ }^{\dagger}$ and C. Verhoeven ${ }^{\ddagger}$ \\ July 7, 2018
}

\begin{abstract}
We consider an integrable scalar partial differential equation (PDE) that is second order in time. By rewriting it as a system and applying the Wahlquist-Estabrook prolongation algebra method, we obtain the zero curvature representation of the equation, which leads to a Lax representation in terms of an energy-dependent Schrödinger spectral problem of the type studied by Antonowicz and Fordy. The solutions of this PDE system, and of its associated hierarchy of commuting flows, display weak Painlevé behaviour, i.e. they have algebraic branching. By considering the travelling wave solutions of the next flow in the hierarchy, we find an integrable perturbation of the case (ii) Hénon-Heiles system which has the weak Painlevé property. We perform separation of variables for this generalized Hénon-Heiles system, and describe the corresponding solutions of the PDE.
\end{abstract}

\section{Introduction}

Recently one of us has been classifying integrable 1+1-dimensional scalar partial differential equations (PDEs) that are second order in time 34, including those of the type

$$
w_{t t}=w_{n x, t}+F\left[w, w_{t}, w_{x}, w_{x t}, \ldots\right]
$$

(where $F$ is a nonlinear function of $w$ and its $x$ and $t$ derivatives). This large class of scalar PDEs includes the Boussinesq equation

$$
u_{t t}=u_{4 x}+\left(u^{2}\right)_{x x}
$$

a well known integrable equation in shallow water wave theory. In the course of performing the classification, the equation

$$
w_{t t}=w_{3 x, t}+8 w_{x} w_{x t}+4 w_{x x} w_{t}-2 w_{x} w_{4 x}-4 w_{x x} w_{3 x}-24 w_{x}^{2} w_{x x} .
$$

was found. The above equation was obtained by means of the perturbative symmetry approach to the classification of integrable PDEs, as presented in [33. In the latter approach, the existence of infinitely many commuting symmetries is taken as the defining property of

${ }^{*}$ Institute of Mathematics, Statistics \& Actuarial Science (IMSAS), University of Kent, Canterbury CT2 7NF, UK. E-mail: anwh@kent.ac.uk

${ }^{\dagger}$ IMSAS, Kent, as above. E-mail: vn9@kent.ac.uk

${ }^{\ddagger}$ Dienst Theoretische Natuurkunde, Vrije Universiteit Brussel, Pleinlaan 2, B-1050 Brussels, Belgium. E-mail: cverhoev@vub.ac.be 
an integrable PDE, and by representing a PDE such as (1.1) symbolically (in Fourier space) one obtains an infinite sequence of necessary conditions for integrability. The equation (1.2) was isolated by the requirement that it should satisfy the first few of these conditions.

Obviously, checking a finite number of necessary conditions does not prove integrability. However, in practice it can be proved a posteriori that only the first few conditions are sufficient for integrability within a given class of equations. In any case, having obtained a particular equation such as (1.2) in the course of classifying the general class of PDEs (1.1), its integrability must then be proved constructively, either by demonstrating that it is explicitly linearizable, or by showing that it can be derived as the compatibility condition for a linear system (Lax pair) of the form

$$
\mathcal{L} \psi=0, \quad \psi_{t}=\mathcal{A} \psi,
$$

for suitable operators $\mathcal{L}, \mathcal{A}$ having coefficients dependent on $w$ and its derivatives, and on a spectral parameter $\lambda$. Once a Lax pair (or, alternatively, a linearization) is known, then the infinite hierarchy of symmetries of an integrable PDE can be obtained directly. To look for a Lax pair, it is most convenient to try to obtain the PDE (1.2) in the form of a zero curvature equation

$$
\mathbf{F}_{t}-\mathbf{G}_{x}+[\mathbf{F}, \mathbf{G}]=0
$$

this being the compatibility condition for the matrix linear system

$$
\Psi_{x}=\mathbf{F} \Psi, \quad \Psi_{t}=\mathbf{G} \Psi
$$

(where the matrices $\mathbf{F}, \mathbf{G}$ are functions of $u$ and its derivatives, and of a spectral parameter).

In the next section we apply the Wahlquist-Estabrook prolongation algebra method to the PDE (1.2) in order to obtain a zero curvature representation for it. The calculations are much facilitated by setting $u=w_{x}$ and $v=w_{t}-w_{3 x}-3 w_{x}^{2}$ and rewriting the scalar equation (1.2) as a two-component system (see (2.1) below). We find that the simplest zero curvature representation for the system (2.1) has $\mathbf{F}, \mathbf{G} \in \mathrm{sl}(2)$, and this is equivalent to a scalar Lax pair (1.3) with the Lax operator being the energy-dependent Schrödinger operator

$$
\mathcal{L}=\lambda \partial_{x}^{2}+\left(\frac{v}{4}+u \lambda-\lambda^{2}\right)
$$

which is an example of the general type

$$
\mathcal{L}=\sum_{j=0}^{N} \lambda^{j}\left(\epsilon_{j} \partial_{x}^{2}+u_{j}\right)
$$

studied by Antonowicz and Fordy [7, 8]. Some particular examples of energy-dependent Schrödinger hierarchies were considered earlier by Jaulent and Miodek 28] and Martinez Alonso 30. (see also the more recent work of Shabat and Martinez Alonso 31]). The Lax pair for (1.2) allows us to write down its hierarchy of higher symmetries. Subsequent sections concern the weak Painlevé property for the PDE (section 3), and travelling wave solutions of members of the hierarchy (section 4). It turns out that the stationary flow of the next member of the hierarchy is equivalent to an integrable perturbation of the case (ii) HénonHeiles system; this extends a result of Fordy concerning the fifth order KdV equation [18. We briefly remark on similarity reductions in our conclusions (section 5). 


\section{Prolongation algebra and integrable hierarchy}

A Lax pair for the scalar PDE (1.2) can be found in a straighforward way by first writing it in the form of a two-component system, that is

$$
\begin{aligned}
& u_{t}=u_{x x x}+6 u u_{x}+v_{x}, \\
& v_{t}=4 u_{x} v+2 u v_{x},
\end{aligned}
$$

in which form the reduction $v=0$ to the $\mathrm{KdV}$ equation

$$
u_{t}=u_{x x x}+6 u u_{x}
$$

is made manifest. Starting from the above system, we then proceed to apply the prolongation algebra method due to Wahlquist and Estabrook [43] (see also [19] for an excellent exposition).

We start by assuming that the system (2.1) has a zero curvature representation (1.4) where the matrix $\mathbf{F}$ depends only on $u, v$, so $\mathbf{F}=\mathbf{F}(u, v)$, and $\mathbf{G}$ depends on $v$ and derivatives of $u$ of order at most two, so $\mathbf{G}=\mathbf{G}\left(u, u_{x}, u_{x x}, v\right)$ (with the dependence on a spectral parameter being suppressed). Plugging this ansatz into the zero curvature equation and substituting for $u_{t}$ and $v_{t}$ from (2.1) yields

$$
\mathbf{F}_{u}\left(u_{3 x}+6 u u_{x}+v_{x}\right)+F_{v}\left(4 u_{x} v+2 u v_{x}\right)-\mathbf{G}_{v} v_{x}-\sum_{j=0}^{2} \mathbf{G}_{u_{j x}} u_{(j+1) x}+[\mathbf{F}, \mathbf{G}]=0
$$

(where subscripts denote partial derivatives). Reading off the coefficient of $u_{3 x}$ in (2.3), we have immediately that

$$
\mathbf{G}_{u_{x x}}=\mathbf{F}_{u}
$$

so that upon integrating we find

$$
\mathbf{G}\left(u, u_{x}, u_{x x}, v\right)=\mathbf{F}_{u} u_{x x}+\hat{\mathbf{G}}\left(u, u_{x}, v\right)
$$

for some matrix function $\hat{\mathbf{G}}$. Substituting back for $\mathbf{G}$ from (2.4) into (2.3), we can then compare coefficients of $u_{x x}$, and we can continue in this way until we obtain explicit expressions for $\mathbf{F}$ and $\mathbf{G}$ as differential polynomials in $u, v$ and their derivatives with matrix coefficients. These constant coefficients must further satisfy certain algebraic relations, including commutators, and for the Lax pair to exist it is necessary that there should be a non-trivial realization of these relations within a suitable Lie algebra. With the substitution of (2.4) and subsequent integrations and back substitutions into (2.3), we find that $\mathbf{F}$ takes the form

$$
\mathbf{F}=\mathbf{A} u^{2}+\mathbf{B} u+\mathbf{C} v+\mathbf{D}
$$

where the matrices $\mathbf{A}, \mathbf{B}, \mathbf{C}, \mathbf{D}$ are constants. However, we note that from the relations between $\mathbf{A}$ and the other coefficients of $\mathbf{F}$ and $\mathbf{G}$, we can consistently set $\mathbf{A}=0$, so that $\mathbf{F}$ is linear in $u$ and $v$. Having made this simplification, we find that $\mathbf{G}$ has the form

$$
\mathbf{G}=\mathbf{B} u_{x x}+[\mathbf{D}, \mathbf{B}] u_{x}+(\mathbf{B}+2 u \mathbf{C}) v+(6 \mathbf{B}-[\mathbf{B},[\mathbf{B}, \mathbf{D}]]) \frac{u^{2}}{2}+[\mathbf{D},[\mathbf{D}, \mathbf{B}]] u+\mathbf{E} .
$$

The relations that must be satisfied by the coefficients are as follows:

$$
[\mathbf{B}, \mathbf{C}]=0, \quad 2 \mathbf{C}+[\mathbf{C},[\mathbf{D}, \mathbf{B}]]=0, \quad(\operatorname{ad} \mathbf{B})^{3} \mathbf{D}=0
$$




$$
\begin{gathered}
{\left[\mathbf{C},(\operatorname{ad} \mathbf{B})^{2} \mathbf{D}\right]=0, \quad\left[\mathbf{B},(\operatorname{ad} \mathbf{D})^{2} \mathbf{B}\right]+\frac{1}{2}\left[\mathbf{D}, 6 \mathbf{B}-(\operatorname{ad} \mathbf{B})^{2} \mathbf{D}\right]=0} \\
2[\mathbf{D}, \mathbf{C}]+\left[\mathbf{C},(\operatorname{ad} \mathbf{D})^{2} \mathbf{B}\right]=0, \quad[\mathbf{B}, \mathbf{E}]+(\operatorname{ad} \mathbf{D})^{3} \mathbf{B}=0 \\
{[\mathbf{D}, \mathbf{B}]+[\mathbf{C}, \mathbf{E}]=0, \quad[\mathbf{D}, \mathbf{E}]=0 .}
\end{gathered}
$$

We now seek to realize the above relations non-trivially in a suitable Lie algebra. To begin with we suppose that

$$
\mathbf{C}=\xi \mathbf{B}
$$

for some scalar $\xi$, so that the first relation (2.5) is satisfied, while the second one implies that

$$
[\mathbf{K}, \mathbf{B}]=2 \mathbf{B}
$$

where we have introduced

$$
\mathbf{K}=[\mathbf{D}, \mathbf{B}] .
$$

The relation (2.10) already suggests that $\mathbf{B}$ and $\mathbf{K}$ should be two of the three basis elements of an $\mathrm{sl}(2)$ subalgebra.

If we now consider the third relation in (2.5) then we see that

$$
(\operatorname{ad} \mathbf{B})^{3} \mathbf{D}=-(\operatorname{ad} \mathbf{B})^{2} \mathbf{K}=[\mathbf{B}, 2 \mathbf{B}]=0,
$$

using (2.10), so this relation is automatically satisfied given the assumption (2.9). Furthermore, using (2.9) and (2.10) we see that both relations (2.6) and the first relation (2.7) also hold automatically. Let us now consider the first equations in (2.8), which becomes

$$
[\mathbf{B}, \xi \mathbf{E}-\mathbf{D}]=0 \text {. }
$$

The above obviously holds if we choose to set

$$
\mathbf{E}=\xi^{-1} \mathbf{D}
$$

in which case the second relation (2.8) is trivially true. Finally, we have the second relation (2.7) still to be satified, which becomes

$$
-\xi^{-1} \mathbf{K}+[\mathbf{D},[\mathbf{D}, \mathbf{K}]]=0
$$

Adding a multiple of $\mathbf{B}$ to $\mathbf{D}$ does not change the commutation relation (2.11), so we set

$$
\mathbf{D}=-\mathbf{J}-\lambda \mathbf{B}
$$

for some constant $\lambda$, and then we find it is consistent to take $\mathbf{J}$ and $\mathbf{B}$ as the Chevalley generators of an $\operatorname{sl}(2)$ algebra, so that

$$
[\mathbf{B}, \mathbf{J}]=\mathbf{K}, \quad[\mathbf{K}, \mathbf{B}]=2 \mathbf{B}, \quad[\mathbf{K}, \mathbf{J}]=-2 \mathbf{J},
$$

and the relation (2.12) is satisfied provided that the constants $\xi$ and $\lambda$ are related according to

$$
\xi=\frac{1}{4 \lambda} \text {. }
$$

With the above choices we see that the final expressions for $\mathbf{F}$ and $\mathbf{G}$ take the form

$$
\mathbf{F}=\left(u-\lambda+\frac{v}{4 \lambda}\right) \mathbf{B}-\mathbf{J}
$$


and

$$
\mathbf{G}=\left(u_{x x}+2 u^{2}+v+2 u \lambda-4 \lambda^{2}+\frac{u v}{2 \lambda}\right) \mathbf{B}+u_{x} \mathbf{K}-(2 u+4 \lambda) \mathbf{J} .
$$

If we then choose the standard matrix representation of $\operatorname{sl}(2)$ then $\mathbf{F}$ is just

$$
\mathbf{F}=\left(\begin{array}{cc}
0 & u-\lambda+\frac{v}{4 \lambda} \\
-1 & 0
\end{array}\right)
$$

and then setting $\Psi=\left(-\psi_{x}, \psi\right)^{T}$ we see that the $x$ part of the matrix linear system (1.5) is equivalent to the energy-dependent Schrödinger equation

$$
\frac{1}{\lambda} \mathcal{L} \psi \equiv\left(\partial_{x}^{2}+\frac{v}{4 \lambda}+u-\lambda\right) \psi=0
$$

for $\psi$, with the operator $\mathcal{L}$ as in (1.6). Similarly, the $t$ part of (1.5) implies that the time derivative of $\psi$ is given by

$$
\partial_{t} \psi=(2 u+4 \lambda) \psi_{x}-u_{x} \psi
$$

Very recently, we learnt that Shabat also derived the system (2.1) as a reduction of the so called universal solitonic hierarchy; see the unnumbered equation half way down p. 622 in 38 .

The construction of integrable hierarchies associated with energy-dependent Schrödinger operators of the general type (1.7) was described in detail by Antonowicz and Fordy 6, 7, 8, . We now present the explicit form of their construction for the particular linear problem (2.13), showing how this leads to the hierarchy of commuting flows for the system (2.1), that correspond to the symmetries of the scalar PDE (1.2). Starting from the Schrödinger equation (2.13), the sequence of compatible linear evolution equations for the wave function $\psi$ takes the form

$$
\partial_{t_{2 N+1}} \psi=\left(2 P_{N} \partial_{x}-P_{N, x}\right) \psi \equiv \mathcal{A} \psi
$$

for integers $N=0,1,2, \ldots$ that label the flows, where $P_{N}$ are certain polynomials in $\lambda$ whose coefficients are functions of $u, v$ and their derivatives. For each $N$, the requirement of compatibility between the time evolution (2.15) and the Schrödinger equation (2.13) leads to the generalized Lax equation

$$
\partial_{t_{2 N+1}} \mathcal{L}=[\mathcal{A}, \mathcal{L}]+4 P_{N, x} \cdot \mathcal{L}
$$

which means that the potential of the Schrödinger operator $\mathcal{L}$ must evolve according to

$$
\partial_{t_{2 N+1}} W=\left(\partial_{x}^{3}+4 W \partial_{x}+2 W_{x}\right) P_{N}, \quad W=\frac{v}{4 \lambda}+u-\lambda .
$$

For each $N$, the equation (2.17) encodes the evolution of $u$ and $v$ with respect to the time $t_{2 N+1}$, but the precise from of each $P_{N}$ must be specified in order to obtain the explicit form of these evolution equations. In fact a compatible sequence of polynomials $P_{N}$, of degree $N$ in $\lambda$ for each integer $N \geq 0$, can be consistently generated by considering the product

$$
\mathcal{P}=\psi \psi^{\dagger}
$$

of two independent solutions of the Schrödinger equation (2.13). It is well known 24] that $\mathcal{P}$ satisfies

$$
\mathcal{P} \mathcal{P}_{x x}-\frac{1}{2} \mathcal{P}_{x}^{2}+2 W \mathcal{P}^{2}+8 \lambda=0
$$

which is known as the Ermakov-Pinney equation [16, 35]; note that we have fixed the Wronskian

$$
\psi_{x} \psi^{\dagger}-\psi \psi_{x}^{\dagger}=4 \sqrt{\lambda}
$$


It is also useful to observe that the differential consequence of (2.18) is a third order linear equation for $\mathcal{P}$, namely

$$
\left(\partial_{x}^{3}+4 W \partial_{x}+2 W_{x}\right) \mathcal{P}=0
$$

We can expand the solution of (2.18) about $\lambda=\infty$, so that

$$
\mathcal{P}=\sum_{j=0}^{\infty} f_{j} \lambda^{-j}, \quad f_{0}=2,
$$

and, having fixed the sign of the leading coefficient $f_{0}$, we find that all subsequent coefficients $f_{j}$ are determined uniquely from the Ermakov-Pinney equation by a recursion of the form $f_{j}=\left(\right.$ differential polynomial in $\left.u, v, f_{k}, k<j\right)=($ differential polynomial in $u, v)$. Then $\mathcal{P}$ serves as a generating function for the sequence of polynomials $P_{N}$ in the sense that

$$
P_{N}=\left(\lambda^{N} \mathcal{P}\right)_{+}=\sum_{j=0}^{N} f_{j} \lambda^{N-j}
$$

The first few of the $f_{j}$ are

$$
f_{0}=2, \quad f_{1}=u, \quad f_{2}=\left(u_{x x}+3 u^{2}+v\right) / 4 .
$$

In order to write down the hierarchy of flows (2.17) explicitly in Hamiltonian form, it is helpful to observe that from (2.19) the coefficients of $\mathcal{P}$ satisfy the recursion

$$
4 \partial_{x} f_{j+1}=\left(\partial_{x}^{3}+4 u \partial_{x}+2 u_{x}\right) f_{j}+\frac{1}{2}\left(2 v \partial_{x}+v_{x}\right) f_{j-1}
$$

for $j=0,1,2, \ldots$. Thus, upon expanding out in powers of $\lambda$, all but the two lowest orders in (2.17) cancel out, leaving the equations

$$
\partial_{t_{2 N+1}}\left(\begin{array}{c}
u \\
v
\end{array}\right)=\mathbf{B}_{0}\left(\begin{array}{c}
\delta_{u} \mathcal{H}_{N+1} \\
\delta_{v} \mathcal{H}_{N+1}
\end{array}\right)=\mathbf{B}_{1}\left(\begin{array}{c}
\delta_{u} \mathcal{H}_{N} \\
\delta_{v} \mathcal{H}_{N}
\end{array}\right)
$$

where

$$
\mathbf{B}_{0}=\left(\begin{array}{cc}
4 \partial_{x} & 0 \\
0 & 16 v \partial_{x}+8 v_{x}
\end{array}\right), \quad \mathbf{B}_{1}=\left(\begin{array}{cc}
\partial_{x}^{3}+4 u \partial_{x}+2 u_{x} & 4 v \partial_{x}+2 v_{x} \\
4 v \partial_{x}+2 v_{x} & 0
\end{array}\right)
$$

and

$$
\delta_{u} \mathcal{H}_{N}=4 \delta_{v} \mathcal{H}_{N+1}=f_{N}
$$

with e.g.

$$
\mathcal{H}_{0}=2 \int u d x, \quad \mathcal{H}_{1}=\frac{1}{2} \int\left(u^{2}+v\right) d x, \quad \mathcal{H}_{2}=\frac{1}{8} \int\left(-u_{x}^{2}+2 u^{3}+2 u v\right) d x .
$$

These conserved quantities are just two-field generalizations of the well known Hamiltonians for the KdV hierarchy, to which they reduce upon setting $v=0$. By the results of Antonowicz and Fordy concerning energy-dependent Schrödinger hierarchies [6, 7, 8, $\mathbf{B}_{0}$ and $\mathbf{B}_{1}$ form a compatible pair of Hamiltonian operators, and the existence of the sequence of functionals $\mathcal{H}_{N}$ satisfying (2.24) is also guaranteed (see e.g. the Lemma on p.468 of [8]).

Thus we see that (2.22) constitutes a bi-Hamiltonian formulation of the integrable hierarchy whose first non-trivial member is the system (2.1). From (2.24) it is clear that the differential polynomials $f_{j}$ are gradients of conserved functionals, and the system (2.1) just corresponds to the $N=1$ flow with $t_{3}=t$. We shall return to this formulation of the flows in section 4 when we consider travelling wave solutions. 


\section{Weak Painlevé property}

The connection between the singularity structure of differential equations and their integrability has been exploited since at least the time of Kowalevksi 29]. Some time later, this connection led Ablowitz, Ramani and Segur to make their conjecture [3] that (up to possible changes of variables) all ordinary differential equations arising as reductions of integrable PDEs should have the Painlevé property, i.e. that all movable singularities of their general solution should be poles. While testing for the Painlevé property is a very useful tool for isolating integrable ODEs and PDEs, it has long been known that insisting on only pole singularities is too strong a requirement for integrability. Indeed, there are many examples of integrable systems which have the weaker property that all movable singularities of the general solution have only a finite number of branches, and this weak Painlevé property was proposed by Ramani et al. [36] as a more general criterion for integrability.

In finite-dimensional classical Hamiltonian mechanics there are many examples of Liouville integrable systems with the weak Painlevé property (see e.g. [1, 2]), including the classical problem of geodesic motion on ellipsoids that was solved by Jacobi [27. In this section we apply the weak Painlevé test of [36] to the PDE system (2.1), before making some general remarks concerning weak Painlevé expansions for integrable hierarchies derived from energy-dependent Schrödinger operators, some of which were considered in [23]. The ODE reductions of the system (2.1) that are considered in the next section provide further examples of integrable finite-dimensional systems with the weak Painlevé property.

For the system (2.1) there are two types of possible expansion around movable singularities. The first type of expansion around a movable (non-characteristic) singular manifold $\phi(x, t)=0$ has algebraic branching, and the leading order terms in this principal balance have the form

$$
u \sim \phi_{t} /\left(2 \phi_{x}\right)+a \phi^{2 / 3}, \quad v \sim \frac{2 a\left(\phi_{x}\right)^{2}}{9 \phi^{4 / 3}}
$$

where $a=a(x, t)$ is an arbitrary function. To find the resonances, where new arbitrary functions appear in the expansion, we substitute

$$
u \sim \phi_{t} /\left(2 \phi_{x}\right)+a \phi^{2 / 3}\left(1+\epsilon_{1} \phi^{r}\right), \quad v \sim \frac{2 a\left(\phi_{x}\right)^{2}}{9 \phi^{4 / 3}}\left(1+\epsilon_{2} \phi^{r}\right),
$$

into (2.1) and consider the leading order linear terms in $\epsilon_{1}$ and $\epsilon_{2}$. This gives a $2 \times 2$ homogeneous linear system for the $\epsilon_{j}$, and the vanishing of the determinant of the associated matrix yields the polynomial equation

$$
9 r^{4}-9 r^{3}-10 r^{2}+8 r=0,
$$

whose roots are the resonance values

$$
r=-1,0,2 / 3,4 / 3 \text {. }
$$

The standard resonance $r=-1$ corresponds to the arbitrary choice of $\phi$, while $r=0$ corresponds to $a$ as in (3.1). From the fact that the other two resonances are also nonnegative, it follows that this expansion corresponds to a principal balance, and the fact that these other resonances are non-integer rational numbers means that the algebraic branching cannot be removed simply by changes of the dependent variables (e.g. choosing $v^{3}$ as a new variable does not remove the cube root branching from the solution).

The resonance conditions at the values in (3.2) are all satisfied, which means that the leading order terms (3.1) can be consistently extended to yield the Puiseux series

$$
u=\phi_{t} /\left(2 \phi_{x}\right)+\sum_{j=0}^{\infty} u_{j} \phi^{2 / 3+j}, \quad v=\sum_{j=0}^{\infty} v_{j} \phi^{-4 / 3+j}
$$


where the singular manifold $\phi$ and the $u_{0}=9 v_{0} / 2=a, u_{2}, u_{4}$ are arbitrarily chosen functions of $x$ and $t$, and all other coefficients $u_{j}$ and $v_{j}$ are determined uniquely in terms of these four functions and their derivatives.

The second type of expansion of the solutions $u, v$ of (2.1) around movable singularities on $\phi(x, t)=0$ has the leading order behaviour

$$
u \sim-\frac{2 \phi_{x}^{2}}{\phi^{2}}, \quad v \sim b \phi^{4}
$$

where $b=b(x, t)$ is arbitrary. In the degenerate case $b=0$ (corresponding to $v=0$ ) this just reduces to the well known expansion for the $\mathrm{KdV}$ equation that was first obtained by the WTC method 44. This balance has resonance values

$$
r=-1,0,4,6
$$

where $r=-1$ is standard, $r=4$ and $r=6$ correspond to arbitrary functions that can be introduced as the coefficients of $\phi^{2}$ and $\phi^{4}$ in the expansion of $u$ (just as in the case of $\mathrm{KdV}$ ). The extra resonance value $r=0$ corresponds to the arbitrary function $b$ appearing at leading order in the expansion of $v$, as in (3.4), and thus we see that this is a second principal balance. Also, the resonance conditions for this expansion are trivially satisfied, and it leads to a standard series of WTC type, namely

$$
u=2(\log \phi)_{x x}+\sum_{j=0}^{\infty} u_{j} \phi^{j}, \quad v=\sum_{j=0}^{\infty} v_{j} \phi^{4+j},
$$

where $\phi$ and the coefficients $v_{0}=b, u_{2}, u_{4}$ may be chosen arbitrarily, with all other coefficients being uniquely determined by them.

It is known that for some integrable systems, there are transformations of hodograph (or reciprocal) type which can be used to transform a PDE with algebraic branching into one with the strong Painlevé property [13, 15, but it is by no means clear that this can always be done. In fact, in the paper [23, one of us made the assertion that reciprocal transformations of a certain type should succeed in removing all algebraic branching from the solutions of certain energy-dependent Schrödinger hierarchies based on Lax operators of the form

$$
\mathcal{L}=\partial_{x}^{2}+\sum_{j=0}^{N} \lambda^{j} u_{j}
$$

This assertion was verified for the principal balances in such hierarchies, but a more careful consideration of non-principal balances suggests that the transformations considered in [23] were actually insufficient to remove all branching from the solutions. Thus the question as to whether PDEs such as (2.1) and the systems treated in 23] admit reciprocal transformations which transform them into PDEs with the strong Painlevé property remains open. In the next section we consider ODE reductions of (2.1) whose general solutions share the same algebraic branching as the original PDE system.

\section{Travelling waves and perturbed Hénon-Heiles}

Here we consider the stationary reductions of the higher order flows of the hierarchy associated with the system of PDEs (2.1). In fact, more generally we will consider travelling wave 
solutions of the higher flows $\partial_{t_{2 N+1}}$ such that the dependence on $x$ and $t_{2 N+1}$ corresponds to waves moving with speed $c$, so that

$$
u=u(z), \quad v=v(z), \quad z=x-c t_{2 N+1}
$$

(where the dependence on the other times $t_{2 j+1}$ has been suppressed). In that case, the travelling wave reduction of the $t_{2 N+1}$ flow takes the form

$$
\begin{aligned}
\left(\partial_{z}^{3}+2\left(u \partial_{z}+\partial_{z} u\right)\right)\left(f_{N}+c / 2\right)+\frac{1}{2}\left(v \partial_{z}+\partial_{z} v\right) f_{N-1} & =0 \\
2\left(v \partial_{z}+\partial_{z} v\right)\left(f_{N}+c / 2\right) & =0
\end{aligned}
$$

The stationary flow corresponds to setting $c=0$.

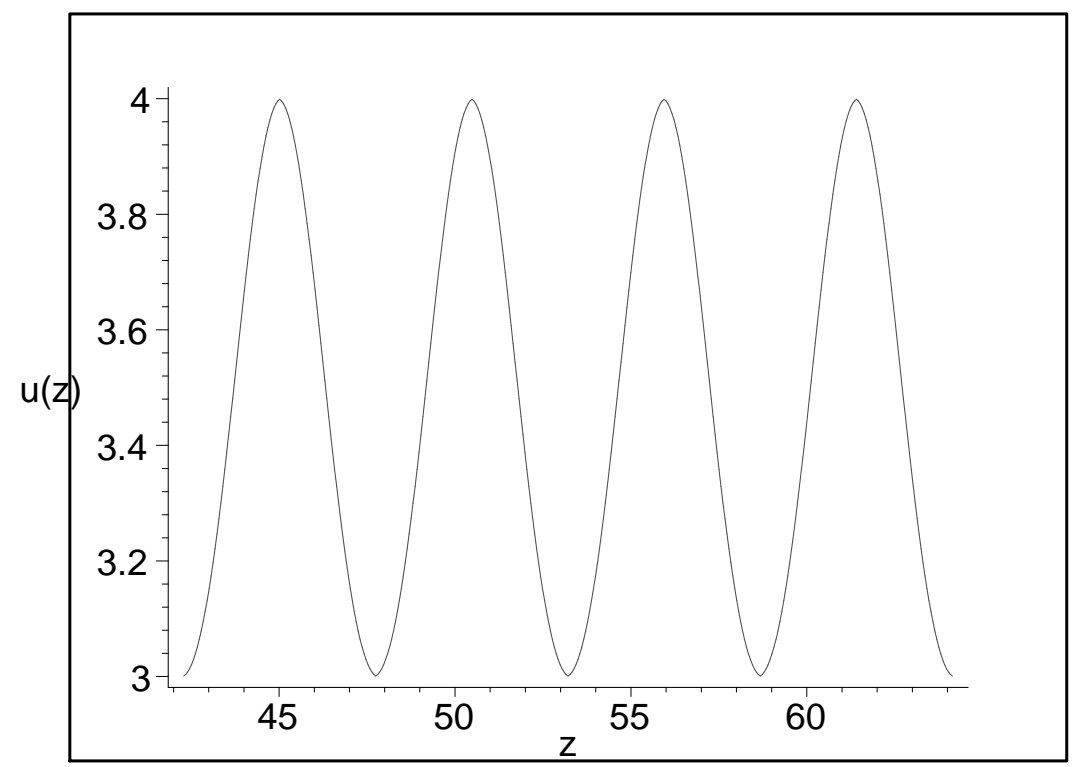

Figure 1: The profile of $u(z)$ for a generic one-phase solution of the system (2.1).

Letting $f=f_{N}+c / 2$ and $g=f_{N-1}$, the second equation (4.1) can be integrated as

$$
v=\frac{K}{f^{2}}
$$

for some constant $K$, and then substituting for $v$ in the first of these equations and integrating again yields the differential equation

$$
f f^{\prime \prime}-\frac{1}{2}\left(f^{\prime}\right)^{2}+2 u f^{2}+\frac{K g}{f}+L=0
$$

where $L$ is another constant.

First we consider the case $N=1$, which corresponds to the travelling wave reduction of the original PDE system (2.1). In that case we have $f_{0}=g=2, f_{1}=u$ so $f=u+c / 2$, and the ODE (4.2) becomes

$$
f f^{\prime \prime}-\frac{1}{2}\left(f^{\prime}\right)^{2}+2(f-c / 2) f^{2}+\frac{2 K}{f}+L=0,
$$

which can be further integrated to yield the quadrature

$$
z-z_{0}=\int^{u+c / 2} \frac{\sqrt{-f} \mathrm{~d} f}{\sqrt{2 \mathcal{Q}(f)}}
$$


where $\mathcal{Q}$ is the quartic polynomial

$$
\mathcal{Q}(f)=f^{4}-c f^{3}-M f^{2}-L f-K \equiv\left(f-e_{1}\right)\left(f-e_{2}\right)\left(f-e_{3}\right)\left(f-e_{4}\right),
$$

and $M, z_{0}$ are two more integration constants. In the affine coordinates $(f, y)$, it can be seen directly that the curve

$$
f y^{2}+2 \mathcal{Q}(f)=0
$$

defines a two-sheeted cover of the Riemann sphere with six branch points, and so has genus two. This can also be seen by making the change of variables

$$
X=-1 / f, \quad Y=y / f^{2},
$$

so that the curve takes the more standard hyperelliptic form

$$
Y^{2}=2 X\left(e_{1} X+1\right)\left(e_{2} X+1\right)\left(e_{3} X+1\right)\left(e_{4} X+1\right),
$$

and the quadrature (4.4) becomes the hyperelliptic integral

$$
z-z_{0}=\int^{-(u+c / 2)^{-1}} \frac{\mathrm{d} X}{Y}
$$

Note that, because the inversion problem (4.7) involves a single integral of one holomorphic differential on a curve of genus two, the simple ODE (4.3) (which can be rewritten in Hamiltonian form) has deficiency one in the terminology of Abenda and Fedorov [1, 2]. This can be seen as the origin of the weak Kowalevski-Painlevé property for the solutions. Nonetheless, it is possible to obtain real solutions without any singularities on the real axis, by choosing suitable initial data to ensure that the quantity $f$ lies between two real branch points, $e_{3} \leq f \leq e_{2}$ say. To produce numerical plots of such solutions, rather than directly evaluating the integral (4.4) and then inverting, we have found it very convenient to do a numerical integration of the first order equation

$$
f^{\prime}= \pm \sqrt{\frac{-2\left(f-e_{1}\right)\left(f-e_{2}\right)\left(f-e_{3}\right)\left(f-e_{4}\right)}{f}}
$$

taking into account the sign changes that occur when $f$ reaches one of the branch points $e_{2}, e_{3}$. We have plotted the profile of $u(z)$ for the particular choice $e_{1}=-2, e_{2}=-3$, $e_{3}=-4, e_{4}=-5$ (see figure 11); this corresponds to travelling waves moving with speed $c=\sum_{j} e_{j}=-14$. We refer to the travelling wave solutions given by (4.4) as "one-phase" solutions, by analogy with the corresponding solutions of $\mathrm{KdV}$ and other soliton equations. For the system (2.1) it is not clear whether some such solutions have a clear interpretation in terms of the periodic spectral problem for the associated energy-dependent Lax operator (1.6), so the common terminology "one-gap" is perhaps not appropriate here.

In the case when two of the roots of the polynomial $\mathcal{Q}(f)$ coincide, the associated hyperelliptic curve acquires a singularity and the genus drops to one; then the quadrature (4.4) can be evaluated in terms of elliptic integrals. Furthermore, if two pairs of roots coincide (say $e_{3}=e_{1}, e_{4}=e_{2}$ ) then the genus of the curve drops to zero, and then the quadrature (4.4) can be written explicitly as

$$
z-z_{0}=\frac{ \pm 1}{\sqrt{2}\left(k_{2}^{2}-k_{1}^{2}\right)}\left[k_{2} \log \left(\frac{k_{2}-\sqrt{k_{1}^{2}+k_{2}^{2}-u}}{k_{2}+\sqrt{k_{1}^{2}+k_{2}^{2}-u}}\right)-k_{1} \log \left(\frac{\sqrt{k_{1}^{2}+k_{2}^{2}-u}-k_{1}}{\sqrt{k_{1}^{2}+k_{2}^{2}-u}+k_{1}}\right)\right]
$$




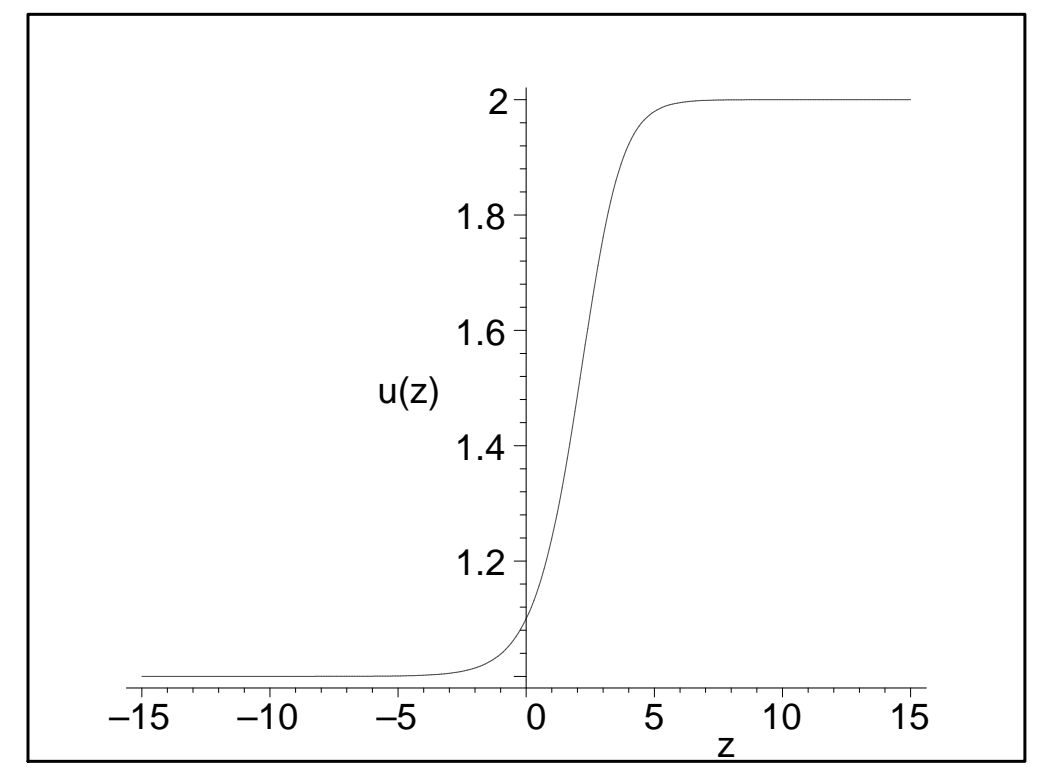

Figure 2: The profile of $u(z)$ for a one-soliton solution of the system (2.1).

where we set

$$
-k_{2}^{2}=e_{2}<e_{1}=-k_{1}^{2}<0 .
$$

The above choice of parameters and branches for the logarithms ensures that there is a real solution $u=f-c / 2$ with $e_{2}<f<e_{1}<0$, so that singularities of the type $f \sim C\left(z-z_{0}\right)^{2 / 3}$ or $f \sim \tilde{C}\left(z-z_{0}\right)^{-2}$ are excluded from the real $z$ axis. This gives a one-soliton solution for the system (2.1), which has a kink shape (like solitons in the sine-Gordon equation). We have plotted an example of such a solution for the choice $e_{1}=-1, e_{2}=-2$, with speed $c=-6$ (figure 21). (Here the term "one-soliton" is used in a general sense, because as far as we are aware the solution does not have a direct interpretation in terms of an inverse scattering transform.)

In the case $N=2$ (the $t_{5}$ flow of the PDE hierarchy) we find that the travelling waves are equivalent to the Hamiltonian system with two degrees of freedom defined by the natural Hamiltonian

$$
h_{1}=\frac{1}{2}\left(p_{1}^{2}+p_{2}^{2}\right)+q_{1}^{3}+\frac{1}{2} q_{1} q_{2}^{2}+2 c q_{1}-\frac{\ell^{2}}{2 q_{2}^{2}}+\frac{256 e q_{1}}{q_{2}^{4}} .
$$

The coordinates $q_{j}$ and momenta $p_{j}$ are canonically conjugate, so $\left\{p_{j}, q_{k}\right\}=\delta_{j k}$, and Hamilton's equations

$$
\frac{\mathrm{d} q_{j}}{\mathrm{~d} z}=\left\{h_{1}, q_{j}\right\}, \quad \frac{\mathrm{d} p_{j}}{\mathrm{~d} z}=\left\{h_{1}, p_{j}\right\}, \quad j=1,2
$$

are equivalent to the ordinary differential equation (4.2) for travelling wave solutions of the $t_{5}$ flow upon setting

$$
u=g=q_{1}, \quad v=\frac{4 e}{f^{2}}=\frac{256 e}{q_{2}^{4}}, \quad f=-q_{2}^{2} / 8=\frac{1}{4}\left(u^{\prime \prime}+3 u^{2}+v+2 c\right) .
$$

The derivation of the equations for $q_{j}, p_{j}$ in Hamiltonian form is omitted, since it is essentially the same as the corresponding calculation for the stationary flow of the fifth order $\mathrm{KdV}$ equation carried out by Fordy [18]; Fordy's results are reproduced by setting $e=0, c=0$.

The equations of motion (4.10) can be written in the form of a Lax equation

$$
\frac{\mathrm{d} \mathbf{L}}{\mathrm{d} z}=[\mathbf{M}, \mathbf{L}]
$$


where the Lax matrix $\mathbf{L}$ is

$$
\mathbf{L}\left(\lambda ; q_{j}, p_{j}\right)=\left(\begin{array}{cc}
-p_{1} \lambda+\frac{1}{4} p_{2} q_{2} & 4 \lambda^{2}+2 q_{1} \lambda-\frac{1}{4} q_{2}^{2} \\
\mathcal{B}\left(\lambda ; q_{j}, p_{j}\right) & p_{1} \lambda-\frac{1}{4} p_{2} q_{2}
\end{array}\right)
$$

with

$$
\mathcal{B}\left(\lambda ; q_{j}, p_{j}\right)=4 \lambda^{3}-2 q_{1} \lambda^{2}+\left(\frac{1}{4} q_{2}^{2}+q_{1}^{2}+2 c\right) \lambda+\frac{1}{4} p_{2}^{2}-\frac{\ell^{2}}{4 q_{2}^{2}}+\frac{128 e q_{1}}{q_{2}^{4}}+\frac{16 e}{q_{2}^{2} \lambda} .
$$

The Lax equation is the compatibility condition for the linear system

$$
\mathbf{L} \Phi=2 \mu \Phi, \quad \frac{\mathrm{d} \Phi}{\mathrm{d} z}=\mathbf{M} \Phi, \quad \mathbf{M}=\left(\begin{array}{cc}
0 & 1 \\
\lambda-q_{1}-\frac{64 e}{q_{2}^{4} \lambda} & 0
\end{array}\right) .
$$

The spectral curve given by $\operatorname{det}(\mathbf{L}-2 \mu \mathbf{1})=0$ has the explicit form

$$
\mu^{2}-4 \lambda^{5}-2 c \lambda^{3}-\frac{1}{2} h_{1} \lambda^{2}-\frac{1}{2} h_{2} \lambda-\frac{\ell^{2}}{64}+\frac{e}{\lambda}=0
$$

with

$$
h_{2}=\frac{1}{4} q_{1} p_{2}^{2}-\frac{1}{4} q_{2} p_{2} p_{1}-\frac{1}{32} q_{2}^{4}-\frac{1}{8}\left(q_{1}^{2}+2 c\right) q_{2}^{2}-\frac{\ell^{2} q_{1}}{4 q_{2}^{2}}+\frac{32 e}{q_{2}^{2}}+\frac{128 e q_{1}}{q_{2}^{4}}
$$

being the second independent integral, in involution with $h_{1}$ i.e. $\left\{h_{1}, h_{2}\right\}=0$. The integral $h_{2}$ generates a second commuting flow

$$
\frac{\mathrm{d} q_{j}}{\mathrm{~d} y}=\left\{h_{2}, q_{j}\right\}, \quad \frac{\mathrm{d} p_{j}}{\mathrm{~d} y}=\left\{h_{2}, p_{j}\right\}, \quad j=1,2 .
$$

Using the relations (4.11) for $u$ and $v$, the joint solutions $q_{j}(z, y), p_{j}(z, y)$ of the pair of compatible Hamiltonian systems defined by $h_{1}$ and $h_{2}$ yield solutions of the PDE system (2.1) upon identifying the independent variables $z=x-c t_{5}, y=4 t=4 t_{3}$.

At this point we should comment on the integrable Hamiltonian system defined by (4.9). Homogeneous Hamiltonians of Hénon-Heiles type were previously classified by using the usual (strong) Painlevé test, requiring meromorphic solutions [12, so it is not surprising that extensions of such potentials with the weak Painlevé property do not appear to have been described in detail before. However, for systems with two degrees of freedom, an extensive search of natural Hamiltonian systems with a second invariant has been performed by Hietarinta [21, and he considered integrable cases of such systems with homogeneous cubic potentials, as well as various perturbations of such systems. The potential (4.9) with a perturbation of the form $q_{1} / q_{2}^{4}$ is a special case of equation (3.2.15) in 21], and can be obtained by making a particular choice of the functions $f$ and $g$ there.

In order to reduce the perturbed Hénon-Heiles Hamiltonian system to quadratures, we perform separation of variables, which we now describe. The separation of variables for the original three integrable cases of the Hénon-Heiles system (with only cubic and harmonic terms in the potential) was obtained in [11] (see also [10]). The generalized integrable cases (i) and (iii), which Fordy had shown to be reductions of the Sawada-Kotera and KaupKupershmidt equations respectively [19], were only separated quite recently 42. As already mentioned, the Hamiltonian (4.9) that we consider here is a perturbation of the integrable case (ii) of the Hénon-Heiles system, and so the separation of variables is straightforward. A new set of canonically conjugate coordinates and momenta $\lambda_{j}, \pi_{j}$ can be obtained according to a well known ansatz (see [39]) whereby the coordinates $\lambda_{1}, \lambda_{2}$ are given by the zeros of the upper right hand entry of the Lax matrix (4.12), so we set

$$
4 \lambda^{2}+2 q_{1} \lambda-\frac{1}{4} q_{2}^{2}=4\left(\lambda-\lambda_{1}\right)\left(\lambda-\lambda_{2}\right)
$$


to find

$$
\lambda_{1}+\lambda_{2}=-\frac{q_{1}}{2}, \quad \lambda_{1} \lambda_{2}=-\frac{q_{2}^{2}}{16} .
$$

In order to find the canonically conjugate momenta, we can write down the generating function

$$
G=-2 p_{1}\left(\lambda_{1}+\lambda_{2}\right)+4 p_{2} \sqrt{-\lambda_{1} \lambda_{2}}, \quad q_{j}=\frac{\partial G}{\partial p_{j}}, \quad \pi_{j}=\frac{\partial G}{\partial \lambda_{j}}, \quad j=1,2,
$$

which means that $p_{j}$ are expressed in terms of the new coordinates and momenta as

$$
\left(\begin{array}{l}
p_{1} \\
p_{2}
\end{array}\right)=\frac{\sqrt{-\lambda_{1} \lambda_{2}}}{2\left(\lambda_{1}-\lambda_{2}\right)}\left(\begin{array}{cc}
-\lambda_{1} / \sqrt{-\lambda_{1} \lambda_{2}} & \lambda_{2} / \sqrt{-\lambda_{1} \lambda_{2}} \\
1 & -1
\end{array}\right)\left(\begin{array}{l}
\pi_{1} \\
\pi_{2}
\end{array}\right) .
$$

Using the above formulae, we can also rewrite $\pi_{1}$ and $\pi_{2}$ in terms of the original variables $q_{j}, p_{j}$, but this will not be necessary for what follows.

Once we are equipped with the expressions (4.15) and (4.16), we can rewrite the two Hamiltonian functions $h_{1}$ and $h_{2}$ in terms of the new variables $\lambda_{j}, \pi_{j}$. Then one can eliminate between these two equations to obtain two separated equations

$$
\frac{\lambda_{j}^{2} \pi_{j}^{2}}{16}-4 \lambda_{j}^{5}-2 c \lambda_{j}^{3}-\frac{1}{2} h_{1} \lambda_{j}^{2}-\frac{1}{2} h_{2} \lambda_{j}-\frac{\ell^{2}}{64}+\frac{e}{\lambda_{j}}=0, \quad j=1,2 .
$$

Upon comparing the equation (4.14) for the spectral curve with the two separated equations (4.17), then with $\pi_{j}=4 \mu_{j} / \lambda_{j}$ the pair of points $\left(\lambda_{j}, \mu_{j}\right)$ for $j=1,2$ lie on the spectral curve. Now we can write down the action $S\left(h_{1}, h_{2} ; \lambda_{1}, \lambda_{2}\right)$ such that

$$
\pi_{j}=\frac{\partial S}{\partial \lambda_{j}}, \quad z_{j}=\frac{\partial S}{\partial h_{j}}, \quad j=1,2
$$

by setting

$$
S=\int^{\lambda_{1}} \frac{4 \mu d \lambda}{\lambda}+\int^{\lambda_{2}} \frac{4 \mu d \lambda}{\lambda}
$$

This yields the quadratures

$$
z_{1}=\int^{\lambda_{1}} \frac{\lambda d \lambda}{\mu}+\int^{\lambda_{2}} \frac{\lambda d \lambda}{\mu}, \quad z_{2}=\int^{\lambda_{1}} \frac{d \lambda}{\mu}+\int^{\lambda_{2}} \frac{d \lambda}{\mu}
$$

with

$$
z_{1}=z+\text { constant, } \quad z_{2}=y+\text { constant. }
$$

The inversion problem (4.19) associated with the hyperelliptic curve (4.14) of genus three involves only two out of three independent holomorphic differentials. This can be augmented with the corresponding relation in terms of a third holomorphic differential,

$$
z_{3}=\int^{\lambda_{1}} \frac{d \lambda}{\lambda \mu}+\int^{\lambda_{2}} \frac{d \lambda}{\lambda \mu},
$$

and then the extra variable is a function of the other two, i.e. $z_{3}=z_{3}\left(z_{1}, z_{2}\right)$. So this perturbed Hénon-Heiles system is another example of an integrable system with deficiency one, and instead of the standard Jacobi inversion involving the threefold symmetric product of the curve, one has the twofold product corresponding to pairs of points $\left(\lambda_{1}, \mu_{1}\right),\left(\lambda_{2}, \mu_{2}\right)$. The image $\left(z_{1}, z_{2}, z_{3}\right)$ of a pair of points under the Abel map lies on a stratum in the Jacobian 


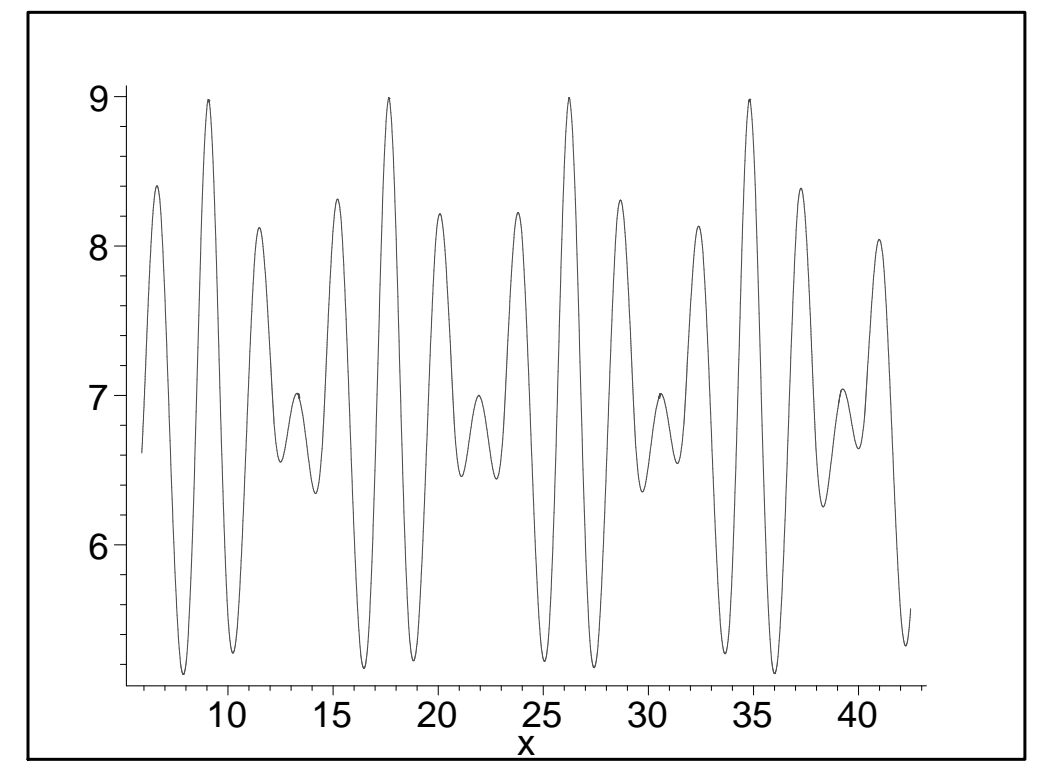

Figure 3: The generic shape of the profile $u(x)=u(x, T)$ for a two-phase solution of the system (2.1), at fixed time $T$.

of the curve, and this leads to an explanation for the algebraic branching in the solutions 1].

The solutions of the perturbed Hénon-Heiles system produce two-phase solutions of the PDE system (2.1), which are the analogues of the two-gap solutions for KdV. The solutions corresponding to higher stationary flows (and travelling waves) of the PDE hierarchy are included in a general ansatz for $N$-phase solutions (see [38], for instance). One can set

$$
\hat{\mathcal{P}}=\psi \psi^{\dagger}=\prod_{j=1}^{N}\left(\lambda-\lambda_{j}\right)
$$

where $\psi, \psi^{\dagger}$ are two independent solutions of the Schrödinger equation (2.13) whose Wronskian is

$$
\psi_{x} \psi^{\dagger}-\psi \psi_{x}^{\dagger}=\mu
$$

with $\mu=\mu(\lambda)$ being given by the equation for the spectral curve,

$$
\mu^{2}=4 \lambda^{2 N+1}+d \lambda^{2 N}+\ldots-e / \lambda
$$

which is of genus $N+1$. Then $\hat{\mathcal{P}}$ satisfies the Ermakov-Pinney equation

$$
\hat{\mathcal{P}} \hat{\mathcal{P}}_{x x}-\frac{1}{2} \hat{\mathcal{P}}_{x}^{2}+2 W \hat{\mathcal{P}}^{2}+\frac{\mu(\lambda)^{2}}{2}=0
$$

The expressions for $u$ and $v$ in terms of $\lambda_{j}$ (trace formulae) are

$$
u=-d / 4-2 \sum_{j=1}^{N} \lambda_{j}, \quad v=\frac{e}{\prod_{j=1}^{N} \lambda_{j}^{2}} ;
$$

these follow immediately from expanding (4.21) around $\lambda=\infty$ and $\lambda=0$. Similarly, expanding around $\lambda=\lambda_{j}$ yields the Dubrovin equations

$$
\lambda_{j}^{\prime}=\frac{\mu\left(\lambda_{j}\right)}{\prod_{k \neq j}\left(\lambda_{j}-\lambda_{k}\right)}, \quad j=1, \ldots, N
$$




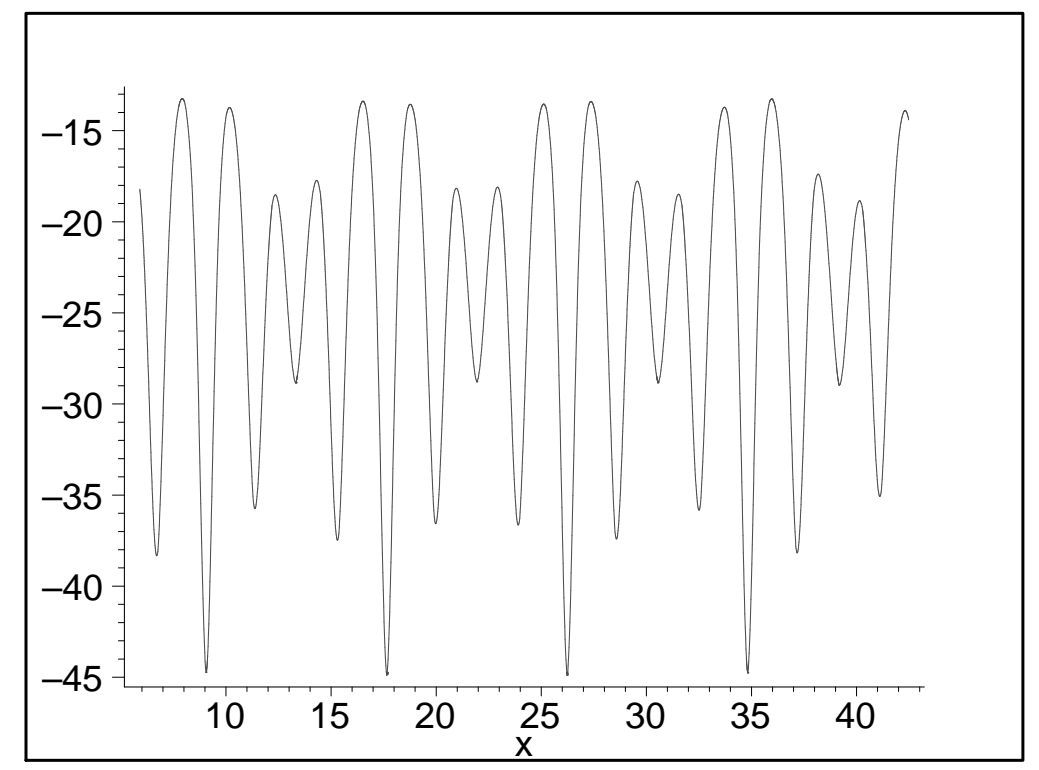

Figure 4: The generic shape of the profile $v(x)=v(x, T)$ for a two-phase solution of the system (2.1), at fixed time $T$.

with prime denoting derivative with respect to $x$ and $\mu$ being a square root of the right hand side of (4.20).

Imposing the time dependence (2.14) upon $\psi$ and $\psi^{\dagger}$ means that $\hat{\mathcal{P}}$ evolves with $t$ according to

$$
\hat{\mathcal{P}}_{t}=(2 u+4 \lambda) \hat{\mathcal{P}}_{x}-2 u_{x} \hat{\mathcal{P}},
$$

and this implies that the time evolution of the roots of the polynomial $\hat{\mathcal{P}}$ is given by

$$
\lambda_{j, t}=-\frac{\left(d / 2+4 \sum_{k \neq j} \lambda_{k}\right) \mu\left(\lambda_{j}\right)}{\prod_{k \neq j}\left(\lambda_{j}-\lambda_{k}\right)}, \quad j=1, \ldots, N .
$$

Just as in the one-phase case, the Dubrovin equations are extremely convenient for obtaining the solutions numerically. We plotted the profile $u(x, t)$ of a two-phase solution at a fixed time $t=T$ by doing a numerical integration of the equations (4.23) for $N=2$ (see figure 3); for this example we placed the branch points of the associated genus three curve at $\lambda=1,2,3,4,5,6$ (as well as the fixed branch points at $\lambda=0, \infty$ ). The corresponding two-phase profile of $v(x, T)$ appears in figure 4.

It is also interesting to consider the two-soliton solutions of (2.1) which arise from the coalescence of three pairs of branch points on the genus three curve. In order to produce plots of such a solution, we performed numerical integration of the Dubrovin equations (4.23) for $N=2$, in the particular case when the right hand side of (4.20) has double roots at $\lambda=1,2,3$, using particular initial conditions $\left.\lambda_{1}\right|_{x=0}=\lambda_{1}\left(0, T_{0}\right),\left.\lambda_{2}\right|_{x=0}=\lambda_{2}\left(0, T_{0}\right)$, with

$$
1<\lambda_{1}\left(0, T_{0}\right)<2, \quad 2<\lambda_{2}\left(0, T_{0}\right)<3
$$

This produced the two-soliton profile of $u\left(x, T_{0}\right)=-2 \lambda_{1}\left(x, T_{0}\right)-2 \lambda_{2}\left(x, T_{0}\right)-d / 4$ with $d=-48$, corresponding to the configuration of two solitons at some fixed time $t=T_{0}$ (plotted in figure 5). In order to observe the subsequent evolution of the pair of solitons at times $t>T_{0}$, we then integrated the two equations (4.24) for $j=1,2$, starting from the initial data $\left.l a_{1}\right|_{t=T_{0}}=\lambda_{1}\left(0, T_{0}\right),\left.l a_{2}\right|_{t=0}=\lambda_{1}\left(0, T_{0}\right)$, in order to get values for $\lambda_{j}(0, t)$ for $t>T_{0}$. For a fixed sequence of times $t=T_{n}$, the values $\lambda_{j}\left(0, T_{n}\right), j=1,2$ were then used as initial data for the Dubrovin equations (4.23), in order to produce the sequence of profiles 


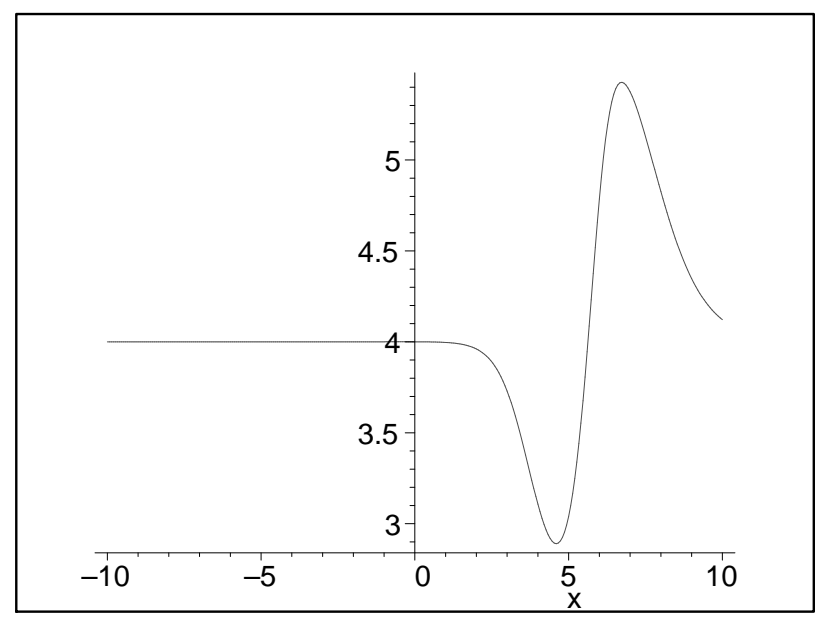

Figure 5: The profile of $u(x)=u\left(x, T_{0}\right)$ for a two-soliton solution of the system (2.1) at time $T_{0}$ (before collision).

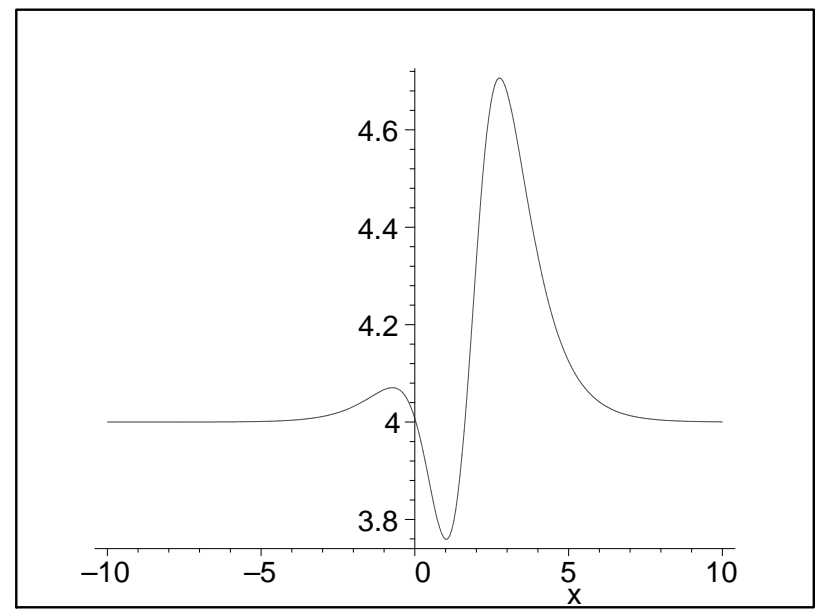

Figure 6: The profile of $u(x)=u\left(x, T_{1}\right)$ for a two-soliton solution of the system (2.1) at time $T_{1}$ (just before collision).

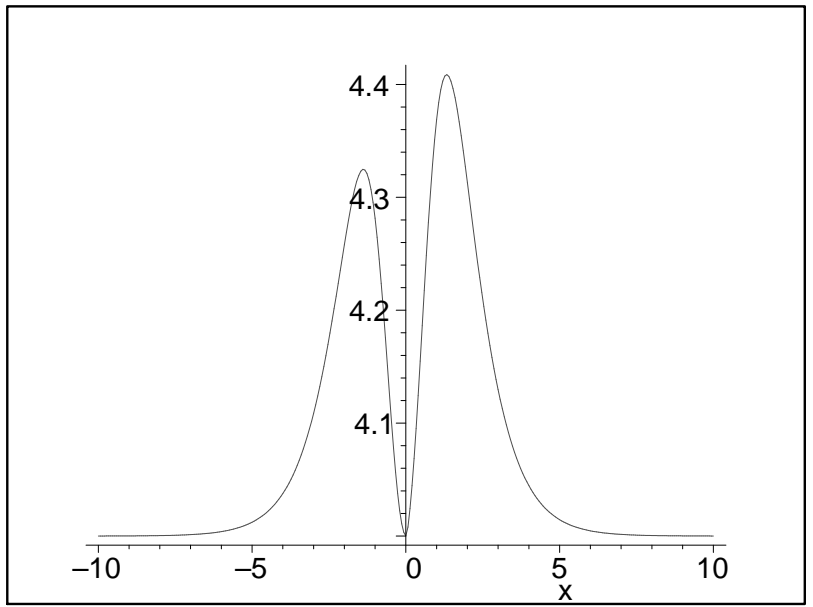

Figure 7: The profile of $u(x)=u\left(x, T_{2}\right)$ for a two-soliton solution of the system (2.1) at time $T_{2}$ (during interaction).

of $u\left(x, T_{n}\right)$. The interaction between the two solitons can be seen in figures [6]- 9] The shape of the field $v(x, t)=e /\left(\lambda_{1}^{2} \lambda_{2}^{2}\right)$ for the two-soliton solution (with $e=-144$ in this example) is 
qualitatively similar to that of $u(x, t)$, but for completeness we have also plotted $v\left(x, T_{2}\right)$ in figure 10, which is at the same stage of the soliton interaction as the corresponding profile of $u$ in figure 7

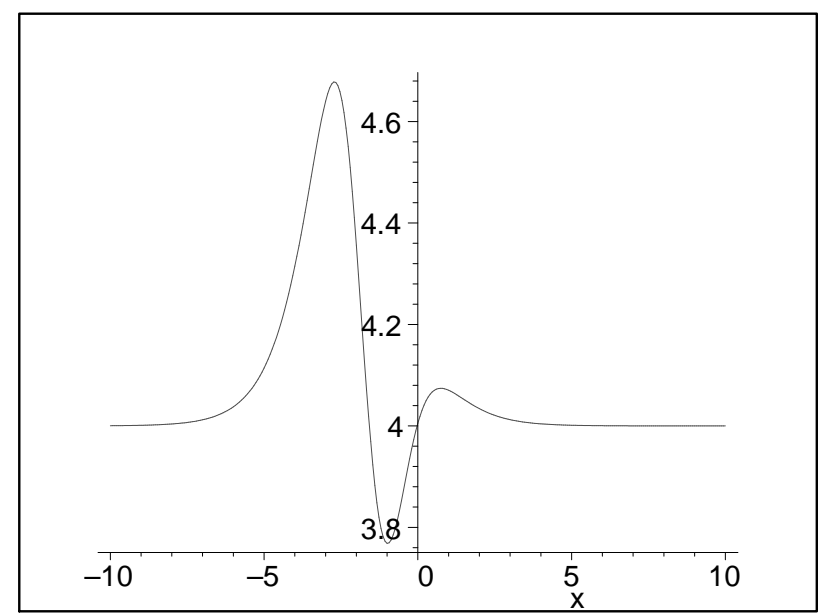

Figure 8: The profile of $u(x)=u\left(x, T_{3}\right)$ for a two-soliton solution of the system (2.1) at time $T_{3}$ (just after collision).

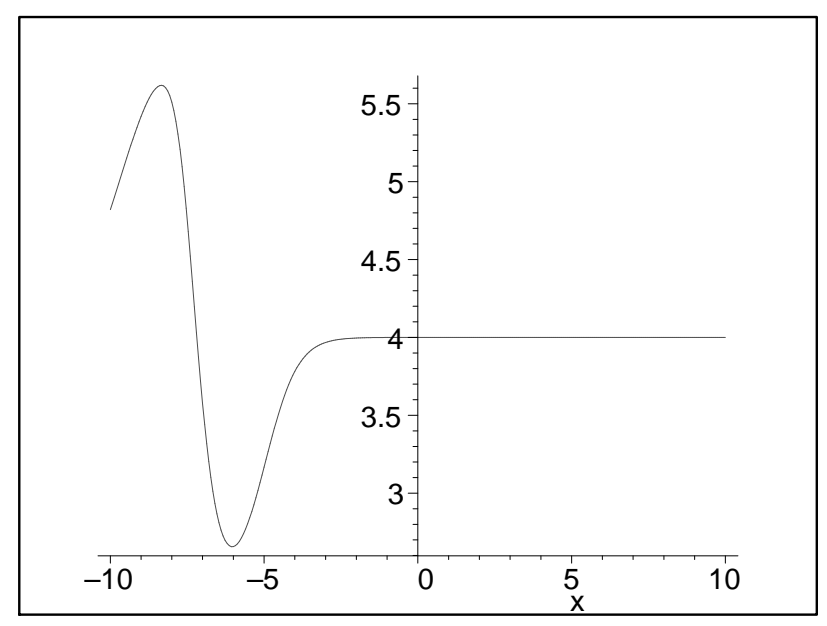

Figure 9: The profile of $u(x)=u\left(x, T_{4}\right)$ for a two-soliton solution of the system (2.1) at a later time $T_{4}$ (after interaction).

\section{Conclusions}

The Wahlquist-Estabrook method has proved to be effective for finding the Lax pair for the PDE (1.2), leading to a $2 \times 2$ zero curvature representation. The latter is equivalent to a scalar Lax pair involving the energy-dependent Schrödinger equation (2.13). The solutions of integrable hierarchies associated with energy-dependent Schrödinger operators have some interesting features compared with those of other integrable systems [4, 5]. As far as we are aware, the theory of inverse scattering has not been developed for such operators (apart from the case where $\mathcal{L}$ is quadratic in $\lambda$, starting with the work [28]).

The solutions of such hierarchies generally display the weak Painlevé property [23], and in particular the algebro-geometric solutions often correspond to finite-dimensional integrable Hamiltonian systems with deficiency [1]. For the $N$-phase solutions of (2.1) described above, 


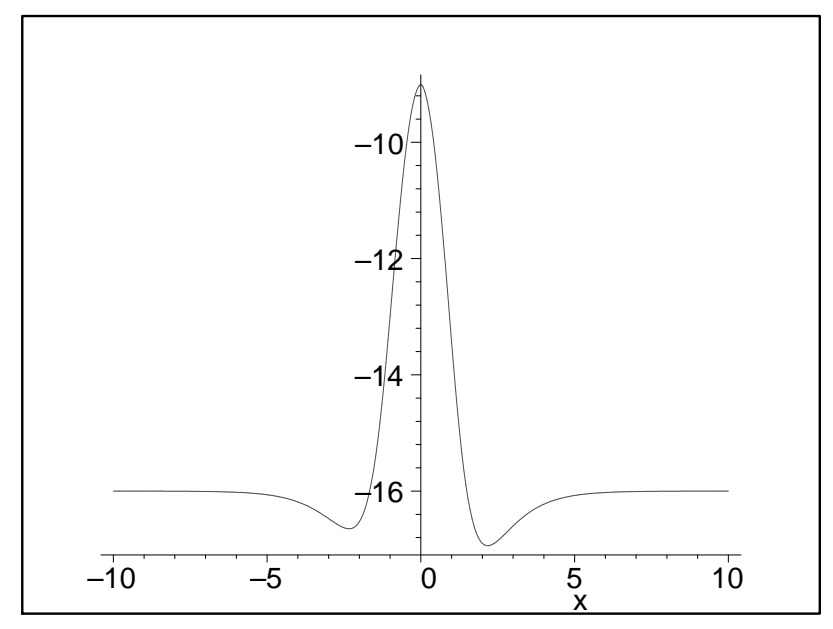

Figure 10: The profile of $v(x)=v\left(x, T_{2}\right)$ for a two-soliton solution of the system (2.1) at time $T_{2}$ (during interaction).

the deficiency is one: the genus of the curve (4.20) is one higher than the number of degrees of freedom. It is interesting to note that, while many such Hamiltonian systems were obtained in 2] by applying Dirac reduction to algebraically completely integrable systems that linearize on a $g$-dimensional Jacobian associated with a genus $g$ curve (with the number of constraints being the deficiency), the systems considered here can be viewed instead as deformations where the genus increases from $g$ to $g+1$ : the parameter $e$ is the deformation parameter, and the spectral curves for the KdV hierarchy are recovered in the limit $e \rightarrow 0$. In particular, the perturbed Hénon-Heiles system with Hamiltonian (4.9) corresponds to a deformation from genus two to genus three. One can obtain multi-parameter deformations (with correspondingly greater deficiency) by considering the Lax operator

$$
\hat{\mathcal{L}}=\partial_{x}^{2}-\lambda+u+\sum_{j=1}^{d} v_{j} \lambda^{-j} .
$$

We have plotted some one- and two-phase solutions of the PDE system (2.1), including their solitonic limiting cases. The soliton dynamics has some quite different features compared with KdV solitons, and is worthy of further study. In the absence of an inverse scattering transform for the PDE system (2.1), one can wonder to what extent the class of soliton-type or $N$-phase solutions represent the general solution. For the case of some integrable PDEs, such as the KdV and nonlinear Schrödinger equations [20], it is possible to make local approximations to arbitrary periodic solutions using finite-gap ones.

Since the first draft of this paper was written, it has been brought to our attention that the Lax pair for the system (2.1) was written down by Zakharov some time ago [45. Ito found the bi-Hamiltonian structure and conservation laws for this system rewritten in the variables $u$ and $V=\sqrt{v}$ [26]. In this form, Matsuno studied the dispersionless limit and also gave an implicit expression for the travelling waves of the system, providing a parametric formula for the quadrature (4.4), or equivalently the hyperelliptic integral (4.7), in terms of Jacobi elliptic functions (see Appendix B in [32]). More recently, the system (2.1) has reappeared in a symmetry classification of mixed scalar and vector evolution equations (see 41], section 3.2.3).

Scaling similarity reductions of integrable PDEs commonly yield non-autonomous ODEs of Painlevé type, and it has been observed that these often inherit the same Hamiltonian structures as their autonomous counterparts (see e.g. [22] and references). However, for scaling similarity reductions of the PDE hierarchy associated with (2.1) this appears not to 
be the case. For example, following the method of Clarkson and Kruskal [14] we see that by setting

$$
u(x, t)=\theta^{2} U(z), \quad v(x, t)=\theta^{4} V(z),
$$

with

$$
z=x \theta(t), \quad \frac{\mathrm{d} \theta}{\mathrm{d} t}=\theta^{4}
$$

(so that $\left.\theta=\left(-3 t+3 t_{0}\right)^{-1 / 3}\right)$, (2.1) is reduced to a coupled system of ODEs, namely

$$
\begin{aligned}
U^{\prime \prime \prime}+6 U U^{\prime}+V^{\prime}-z U^{\prime}-2 U & =0 \\
(2 U-z) V^{\prime}+4\left(U^{\prime}-1\right) V & =0
\end{aligned}
$$

where the prime denotes $\mathrm{d} / \mathrm{d} z$. The integrating factors which allow the ODEs for travelling waves to be written as the single second order equation (4.3) no longer work for this nonautonomous system, except in the special case $V \equiv 0$ when (5.1) reduces to the equation PXXXIV (see [25], chapter XIV). Aside from this special case, the general solution of the fourth order system (5.1) will have the weak Painlevé property. Nevertheless, by applying the technique of Flaschka and Newell [17], we find that putting

$$
\phi(z ; \zeta)=\theta^{2} \psi(x, t ; \lambda), \quad \zeta=\theta^{-2} \lambda
$$

leads to the linear system

$$
\begin{aligned}
\phi^{\prime \prime}+(U-\zeta+V /(4 \zeta)) \phi & =0, \\
\zeta \partial_{\zeta} \phi & =-(2 U-z+4 \zeta) \phi^{\prime}+\left(U^{\prime}-2\right) \phi .
\end{aligned}
$$

The fourth order ODE system arises as the compatibility condition for this linear system, so while (5.1) does not have the Painlevé property it should still be possible to interpret it via isomonodromic deformation of the second equation (5.2).

Another integrable scalar equation of the type (1.1) considered in [34] is equivalent to the system

$$
\begin{aligned}
& u_{t}=u_{5 x}+25 u_{x} u_{x x}+10 u u_{3 x}+20 u^{2} u_{x}+v_{x}, \\
& v_{t}=3 u_{3 x} v+u_{x x} v_{x}+24 u u_{x} v+4 u^{2} v_{x} .
\end{aligned}
$$

The above system is a rewriting of equation number (101) in [34, which is obtained by taking $u=w_{x}$ and eliminating $v$. This system reduces to the Kaup-Kupershmidt equation when $v=0$. By analogy with our results for the system (1.2), related to an energy-dependent operator of second order, it is natural to guess that the system (5.3) should possesses a scalar Lax pair with an energy-dependent Lax operator of third order, which generalizes the Lax pair of the Kaup-Kupershmidt equation. Indeed this turns out to be the case, and we obtain the Lax pair

$$
\begin{aligned}
\frac{1}{\lambda} \mathcal{L} \psi & \equiv\left(\partial_{x}^{3}+2 u \partial_{x}+u_{x}-\frac{v}{9 \lambda}-\lambda\right) \psi=0, \\
\partial_{t} \psi & =-9 \lambda \psi_{x x}+\left(u_{x x}+4 u^{2}\right) \psi_{x}-\left(u_{3 x}+8 u u_{x}+12 u\right) \psi
\end{aligned}
$$

for (5.3). This Lax pair is one member of a family classified by Antonowicz, Fordy and Liu 9]. It is also equivalent to the $3 \times 3$ zero curvature equation recently given in [37. By a slight extension of Fordy's results in [18, we find that the travelling wave reduction of (5.3) corresponds to another perturbed Hénon-Heiles system, with Hamiltonian

$$
h=\frac{1}{2}\left(p_{1}^{2}+p_{2}^{2}\right)+\frac{4}{3} q_{1}^{3}+\frac{1}{4} q_{1} q_{2}^{2}+2 c q_{1}-\frac{\ell^{2}}{2 q_{2}^{2}}+\frac{\tilde{e}}{q_{2}^{6}} .
$$


This reduces to the (generalized) integrable case (iii) Hénon-Heiles system when $\tilde{e}=0$, for $\tilde{e} \neq 0$ it has the weak Painlevé property. The possibility of including the extra term $1 / q_{2}^{6}$ in the potential, while still preserving integrability, was noted some time ago by Hietarinta 21. However, at present we do not know how to carry out separation of variables for this perturbed Hamiltonian system; the results of 42 cannot be directly extended to this case.

Acknowledgements. Andrew Hone acknowledges the support of the EPSRC, and Vladimir Novikov is grateful for support from the GIFT project and from an EPSRC Postdoctoral Fellowship. Caroline Verhoeven is grateful for FWO support in the form of a Postdoctoral Research Fellowship. Andrew Hone is grateful to Yuri Fedorov and Carles Simó for useful remarks during his visit to Barcelona. The authors would also like to thank Jarmo Hietarinta, Takayuki Tsuchida and Sergei Sakovich for valuable comments on the original version of this paper.

\section{References}

[1] Abenda S and Fedorov Y 2000 Acta Appl. Math. 60137.

[2] Abenda S and Fedorov Y 2001 J. Nonlin. Math. Phys. 8 Supplement 1.

[3] Ablowitz M J, Ramani A and Segur H 1978 Lett. Nuovo Cim. 23333.

[4] Alber M S, Luther G G and Marsden J E 1997 Nonlinearity 10223.

[5] Alber M S, Camassa R, Fedorov Yu, Holm DD and Marsden J E 1999 Phys. Lett. A 264171.

[6] Antonowicz A and Fordy A P 1987 Phys. Lett. A 12295.

[7] Antonowicz A and Fordy A P 1987 Physica D 28345.

[8] Antonowicz A and Fordy A P 1989 Commun. Math. Phys. 124465.

[9] Antonowicz A, Fordy A P and Liu Q P 1991 Nonlinearity 4669.

[10] Blaszak M 1998 Multi-Hamiltonian theory of dynamical systems, New York: Springer.

[11] Caboz R, Gavrillov L and Ravoson V 1993 J. Math Phys. 342385.

[12] Chang Y F, Tabor M and Weiss J 1982 J. Math. Phys. 23531.

[13] Clarkson P A, Fokas A S and Ablowitz M J 1989 SIAM J. Appl. Math. 491188.

[14] Clarkson P A and Kruskal M D 1989 J. Math. Phys. 302201.

[15] Degasperis A, Holm D D and Hone A N W 2002 Theoretical and Mathematical Physics 133 1461.

[16] Ermakov V P 1880 Univ. Izv. Kiev Series III 91.

[17] Flaschka H and Newell A C 1980 Commun. Math. Phys. 7665.

[18] Fordy A P 1991 Physica D 52204.

[19] Fordy A P 1990 Prolongation structures of nonlinear evolution equations, in Soliton theory: a survey of results (ed. A.P. Fordy), Manchester University Press, pp. 403-425.

[20] Grinevich P G 2001 Physica D 152-153 20.

[21] Hietarinta J 1987 Physics Reports 14787.

[22] Hone A N W 1998 Physica D 1181. 
[23] Hone A N W 1998 Phys. Lett. A 24946.

[24] Hone A N W 1999 Phys. Lett. A 263347.

[25] Ince E L 1926 Ordinary Differential Equations. Reprint: Dover Publications, New York (1956).

[26] Ito M 1982 Phys. Lett. A 91335.

[27] Jacobi C G 1842 Vorlesungen über Dynamik, Königsberg University, ed. A. Clebsch, Reimer, Berlin (1884).

[28] Jaulent M and Miodek I 1976 Lett. Math. Phys. 1243.

[29] Kowalevski S 1889 Acta Math. 12 177; Kowalevski S 1890 Acta Math. 1481.

[30] Martinez Alonso L 1980 J. Math. Phys. 212342.

[31] Martinez Alonso L and Shabat A B 2002 Phys. Lett. A 299359.

[32] Matsuno Y 2001 J. Math. Phys. 421744.

[33] Mikhailov A V and Novikov V S 2002 J. Phys. A: Math. Gen. 354775.

[34] Mikhailov A V, Novikov V S and Wang J P 2006 On classification of integrable nonevolutionary equations, Studies in Applied Mathematics to appear; nlin.SI/0601046.

[35] Pinney E 1950 Proc. Am. Math. Soc. 1681.

[36] Ramani A, Dorizzi B and Grammaticos G 1982 Phys. Rev. Lett. 491538.

[37] Sergeyev A 2006 Zero curvature representation for a new fifth-order integrable system preprint nlin.SI/0604064.

[38] Shabat A B 2005 J. Nonlin. Math. Phys. 12, Supplement 1614.

[39] Sklyanin E V 1995 Prog. Th. Phys. Supplement 11835.

[40] Tsiganov A V 1999 J. Phys. A: Math. Gen. 327965.

[41] Tsuchida T and Wolf T 2005 J. Phys. A: Math. Gen. 387691.

[42] Verhoeven C, Musette M and Conte R 2002 J. Math. Phys. 431906.

[43] Wahlquist H D and Estabrook F B 1975 J. Math. Phys. 16 1; Wahlquist H D and Estabrook F B 1976 J. Math. Phys. 171293.

[44] Weiss J, Tabor M and Carnevale J 1983 J. Math. Phys. 24522.

[45] Zakharov V E 1980 The inverse scattering method, in Solitons, Bullough R K and Caudrey P J (eds.), Topics in Current Physics vol. 17, pp. 243-285. 